\title{
Requirement for the Candida albicans FAS2 gene for infection in a rat model of oropharyngeal candidiasis
}

\author{
Xiao-Jiong Zhao, ${ }^{1}$ Gail E. McElhaney-Feser, ${ }^{1}$ William H. Bowen, ${ }^{2}$ \\ Michael F. Cole, ${ }^{1}$ Sheldon E. Broedel, $\mathrm{Jr}^{3}$ and Ronald L. Cihlar ${ }^{1}$
}

1 Department of Microbiology and Immunology, Georgetown University, Washington, DC 20007, USA

2 Department of Dental Research, University of Rochester, Rochester, NY, USA

${ }^{3}$ ChekTec Corporation, Baltimore, MD 21227, USA
Author for correspondence: Ronald L. Cihlar. Tel: + 1202687 1136. Fax: +1202687 1800.

e-mail: rcihla01@gumedlib.dml.georgetown.edu

Keywords: Candida albicans, $F A S 2$, null mutants, avirulence, oropharyngeal

\section{INTRODUCTION}

Candida albicans is an opportunistic fungal pathogen of increasing medical significance, particularly in immunocompromised individuals. The organism promotes a wide array of infections ranging from minor irritations to systemic disease (Odds, 1988). Furthermore, C. albicans is responsible for the largest percentage of fungal oropharyngeal infections in the AIDS patient (Holmberg \& Meyer, 1986). A number of potential virulence factors have been identified, including germ-tube formation (Cutler, 1991; De Bernardis et al., 1993), protease production (Ruchel et al., 1992) and phenotypic switching (Soll, 1992). Detailed investigations, however, concerning these processes, as well as analysis of other genes that may specify products required in pathogenesis, have been restricted until recently by the lack of suitable methods to perform certain molecular manipulations. In this regard, development and use of a targeted gene distuption scheme for C. albicans (Fonzi \& Irwin, 1993) has led to the definition of genes required for maximal infectivity
(Ghannoum et al., 1995; Becker et al., 1995), and perhaps more importantly, may lead to identification of promising targets to exploit in the search for new antifungal drugs (Bulawa et al., 1995).

One such potential target is fatty acid synthase. This enzyme directs the synthesis of fatty acids required for the assembly of cellular membrane-containing structures, and is required for cell growth in the absence of fatty acids supplied from an exogenous source. The fungal enzyme comprises two polypeptides, $\alpha$ and $\beta$, specified by the $F A S 2$ and FAS1 genes, respectively (Kuziora et al., 1983; Schweizer et al., 1987). The enzyme is a hexamer and is multifunctional, directing the seven component activities required in fatty acid synthesis (Stoops et al., 1978; Schweizer et al., 1987). In contrast, mammalian fatty acid synthase is a homodimer, with the polypeptide, termed $\alpha$, configured head to tail (Witkowski, et al., 1991 ; Smith, 1994). While homologies exist between fungal and mammalian fatty acid synthases, particularly within catalytic domains (Siggaard-Andersen, 1993), their spacing 
and linear order, as well as the overall structure of the respective enzymes, are clearly distinct (Schweizer $e t$ al., 1987; Smith, 1994). Thus, it is possible that compounds differentially affecting the respective fatty acid synthase might be found, and a recent report has described such agents (Broedel et al., 1996). It remains to be demonstrated, however, that the enzyme is necessary for infection. To address this issue appropriate $C$. albicans FAS2 mutant strains have been constructed and used in experiments examining the virulence of mutants in a rat oral model of candidiasis.

\section{METHODS}

Strains and culture conditions. C. albicans strains used in this study are listed in Table 1 . The strains were maintained on YEPD medium $(1 \%, \mathrm{w} / \mathrm{v}$, yeast extract $; 2 \%, \mathrm{w} / \mathrm{v}$, peptone; $2 \%, w / v$, glucose; and $1.5 \%, w / v$, agar). Defined medium consisted of $2 \%(\mathrm{w} / \mathrm{v})$ glucose supplemented with $0.7 \%(\mathrm{w} / \mathrm{v})$ amino-acid-frce yeast nitrogen base. Media were supplemented with uridine $\left(25 \mu \mathrm{g} \mathrm{ml}^{-1}\right)$ as required. $\mathrm{Ura}^{-}$auxotrophs were sclected on medium containing 5-fluoroorotic acid as described by Boeke et al. (1984). Strain CFD2 (FAS2 null mutant) was grown on YEPD supplemented with $1 \%(\mathrm{v} / \mathrm{v})$ Tween 40 , $0.01 \%(\mathrm{w} / \mathrm{v})$ myristic acid and $0.01 \%(\mathrm{w} / \mathrm{v})$ stearic acid. For growth-curve determination, overnight cultures were grown in YEPD and in some instances were supplemented with fatty acids as above. Subsequently, aliquots were removed, and diluted in fresh medium to an $\mathrm{OD}_{600}$ of $0 \cdot 1$. Cultures were then incubated at $30^{\circ} \mathrm{C}$ on a rotary shaker and, at appropriate time intervals, samples were removed and the $\mathrm{OD}_{600}$ was determined.

DNA manipulations. All restriction endonuclease digestions wete performed under conditions recommended by the manufacturet. Plasmid purification, agarose gel electrophoresis, DNA fragment isolation and Southern blots were performed as described by Sambrook et al. (1989). C. albicans DNA was isolated as reported by Sherman $t$ al. (1986). Probe DNA was labelled with $\left[\alpha{ }^{32} \mathrm{P}\right] \mathrm{dCTP}$ by use of a random prime labelling kit (Stratagene).

Plasmid and strain construction. Plasmid pG10a-1, which contains most of $C$, albicans $F A S 2$ on a $7.9 \mathrm{~kb} S_{a} / \mathrm{I}$ fragment (Southard \& Cihlar, 1995), was digested with Pst and BamHI to release a $2.3 \mathrm{~kb}$ internal fragment of $F . A S 2$ containing the $\beta$ ketoacyl synthase domain (Southard \& Cihlar, 1995). The fragment was recovered following agarose gel electrophoresis and then subcloned into compatible sites of the vector, PUC118.
The resulting plasmid, designated $\mathrm{PCFL}-1$, was digested with EcoRV and $B g l \mathrm{II}$, deleting the condensing reaction domain within a 984 bp fragment. The remaining plasmid fragment was recovered and ligated to a $4.0 \mathrm{~kb} B a m \mathrm{HI}-P \nu u I I$ bisG-UR A3bis $G$ cassette obtained from plasmid p5921 (Fonzi \& Irwin, 1993) to create plasmid pCFU-2. After recovery, pCFU-2 was digested with $P_{s t \mathrm{I}}$ and BamHI releasing a DNA fragment containing the $F A S 2$ gene deletion.

Sequential transformation of strain CAI4 with the constructed cassette was performed as described by Fonzi \& Irwin (1993). First, strain CFD1 was obtained as a single FAS2 allelic disruption by selection of $\mathrm{Ura}^{+}$transformants. Subsequently, a $\mathrm{Ura}^{-}$derivative, $\mathrm{CFD}^{\prime}{ }^{\prime}$ ( $\left.F A S 2 / \Delta f a s 2\right)$ was isolated by selection on medium containing 5-fluoroorotic acid (Boeke et al., 1984). Finally, CFD2 was isolated as a FAS2 null mutant after a second round of transformation with the same cassette. A single copy of the wild-type allele was restored by transformation of strain CFD2 with PstI- and Bg/II-digested pCFU-1. The desired strain, termed CFD3, was then selected on YNB agar plates where growth of either CFD2 or URA ${ }^{-}$colonies was precluded by omission of fatty acid and uridine supplements. The strategy for CFD1 and CFD3 construction ensured that the disruption of FAS2 was not in the same allele in both strains.

Measurement of fatty acid synthase activity. Fatty acid synthase activity was assayed as described elsewhere (Lynen, 1969; McElhaney-Feser \& Cihlar, 1994). Reaction mixtures $(1 \mathrm{ml})$ contained (final concentrations): $0.4 \mathrm{M}$ potassium phosphate, $\mathrm{pH} 7 \cdot 3$, containing $0.125 \mu \mathrm{M}$ dithiothreitol, $50 \mu \mathrm{M}$ acetyl$\mathrm{CoA}, 100 \mu \mathrm{M} \mathrm{NADPH}$, and crude enzyme preparation. Following incubation at $37^{\circ} \mathrm{C}$ for $5 \mathrm{~min}$, malonyl-CoA containing $0.5 \mu \mathrm{Ci} \quad\left[2-{ }^{14} \mathrm{C}\right]$ malonyl-CoA $\quad$ Amersham; $58.8 \mathrm{mCi} \mathrm{mmol}^{-1}$ $\left.\left(2 \cdot 176 \mathrm{GBq} \mathrm{mmol}^{-1}\right)\right]$ was added to a final concentration of $100 \mu \mathrm{M}$. Reactions were terminated after $10 \mathrm{~min}$ by the addition of $30 \mu \mathrm{l} 60 \%(\mathrm{v} / \mathrm{v})$ perchloric acid and $1 \mathrm{ml}$ absolute ethanol. Fatty acids were extracted four times with light petroleum (b.p. $30-75^{\circ} \mathrm{C}$ ), extracts were combined and dried in scintillation vials, and finally solubilized in $3 a 70 B$ scintillation fluid (Research Products International). Radioactivity was measured in a Beckman LSC-230 liquid scintillation counter and enzyme activity was expressed in units ( $\mathrm{mg}$ protein) $)^{-1}$ (1 unit is the amount of enzyme required to catalyse incorporation of $1 \mathrm{nmol}$ $\left[{ }^{14} \mathrm{C}\right]$ malonate into fatty acid $\mathrm{min}^{-1}$ under the conditions of the assay).

Rat model of oropharyngeal candidiasis. The rat model of oropharyngeal candidiasis has been described previously (Meitner et al., 1990; Cole et al., 1995). In the present investigation, weanling, specific pathogen-free Sprague-Dawley

Table 1. C. albicans strains used in this study

\begin{tabular}{|c|c|c|c|}
\hline Strain & Parent & Genotype & Reference \\
\hline SC5314 & & Clinical isolate & $\begin{array}{l}\text { Gillum et al. } \\
\text { (1984) }\end{array}$ \\
\hline CAI4 & SC5314 & 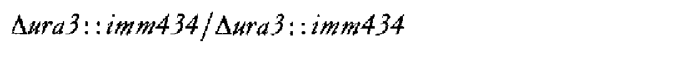 & $\begin{array}{l}\text { Fonzi \& Irwin } \\
\text { (1993) }\end{array}$ \\
\hline CFD1 & CAI4 & $\begin{array}{l}\Delta f a s 2:: \text { bisG-UR A3-bisG/FAS2; } \\
\text { Aura3::imm434/Aura3::imm434 }\end{array}$ & This work \\
\hline CFD1' & CFD1 & $\Delta f a s 2::$ bisG/FAS2; $\Delta a r a 3:: i m m 434 / \Delta u r a 3: ; i m m 434$ & This work \\
\hline CFD2 & CFD1' & $\begin{array}{l}\Delta f a s 2: \text { bisG } / \Delta \text { fas } 2: \text { :bisG-UR } 43-b i s G \\
\text { Aura3::imm } 434 / \Delta u r a 3:: i m m 434\end{array}$ & This work \\
\hline CFD3 & CFD2 & 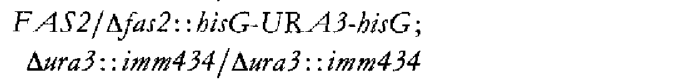 & This work \\
\hline
\end{tabular}


rats, $19-21 \mathrm{~d}$ old, identified by litter, were obtained commercially (Charles River Laboratories). To ensure that animals were free of endogenous $C$. albicans, oral swabs were taken and plated onto YEPD and/or Sabouraud agar. Subsequently, hyposalivation was achieved by ligation of the parotid ducts and removal of the submandibular and sublingual salivary glands.

Oral infection was initiated using a cotton-tipped applicator saturated with $10^{6}$ c.f.u. of an acrively-growing culture of the appropriate $C$. albicans strain. During the course of the experiment rats were fed diet NIH 2000 containing $56 \%(\mathrm{w} / \mathrm{v})$ sucrose, and sucrose $(10 \%, \mathrm{w} / \mathrm{v})$ was added to the drinking water. Animals were killed $7 \mathrm{~d}$ after inoculation. The mandible was then hemisected and removed to $5 \mathrm{ml}$ sterile saline. Adherent organisms were dislodged by sonication, and the level of colonization with $C$. albicans was determined by plating samples obtained from each animal onto Sabouraud agar supplemented with streptomycin $\left(300 \mu \mathrm{g} \mathrm{ml}^{-1}\right)$ or Sabouraud agar supplemented with $1 \%(\mathrm{v} / \mathrm{v})$ Tween $40,001 \%(\mathrm{w} / \mathrm{v})$ stearic acid, $0.01 \%(\mathrm{w} / \mathrm{v})$ myristic acid, and streptomycin $\left(300 \mu \mathrm{g} \mathrm{ml}^{-1}\right)$. Colonies were quantified after incubation of plates overnight at $30^{\circ} \mathrm{C}$.

\section{RESULTS}

\section{C. albicans FAS2 null mutant construction by sequential disruption}

A strategy to construct a homozygous disruptant of $C$. albicans FAS2 was designed utilizing the 'URA-blaster'

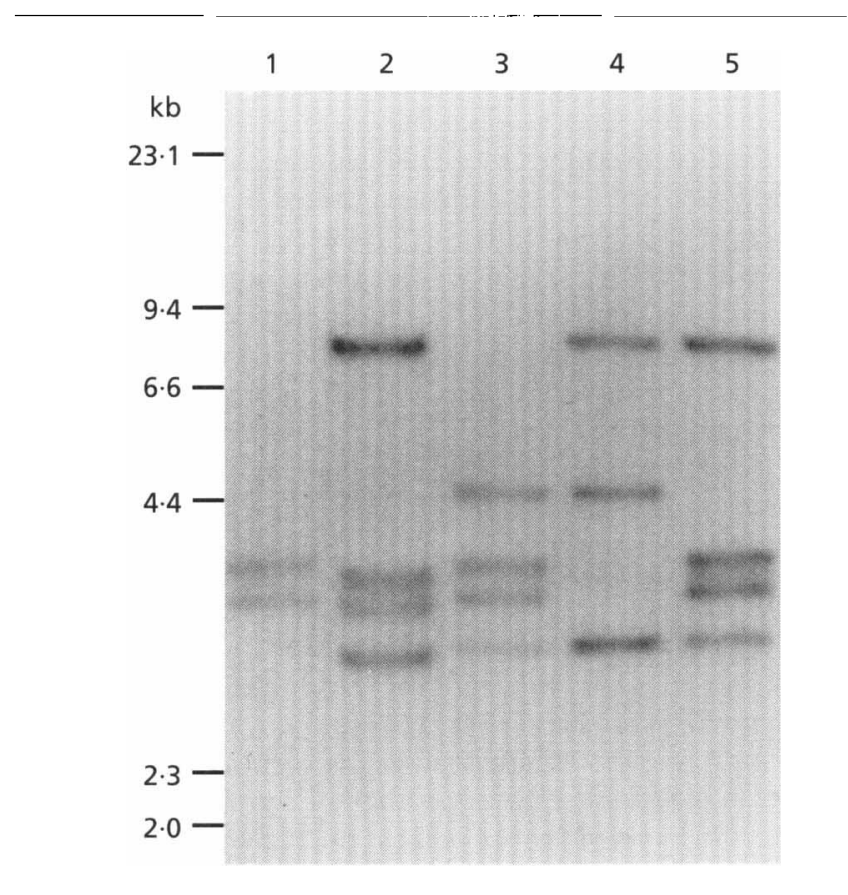

Fig. 1. Southern blot analysis of C. albicans FASZ disrupted/deleted strains. Genomic DNA from individual strains was digested with $B g / l l$, fractionated on a $1 \%$ agarose gel and blotted to a nylon membrane. The filter was hybridized with a $7.9 \mathrm{~kb}$ Sall fragment containing the $C$. albicans FAS2 strain. Genomic DNA from: lane 1, CAI4 (wild-type); lane 2, CFD1 (single FAS2 allele disruption); lane 3, CFD1' (single FAS2 allele deletion; lane 4, CFD2 (FAS2 null mutant, one allele disruption, one allele deletion); lane 5, CFD3 (single FAS2 allele disruption, restoration of second allele).

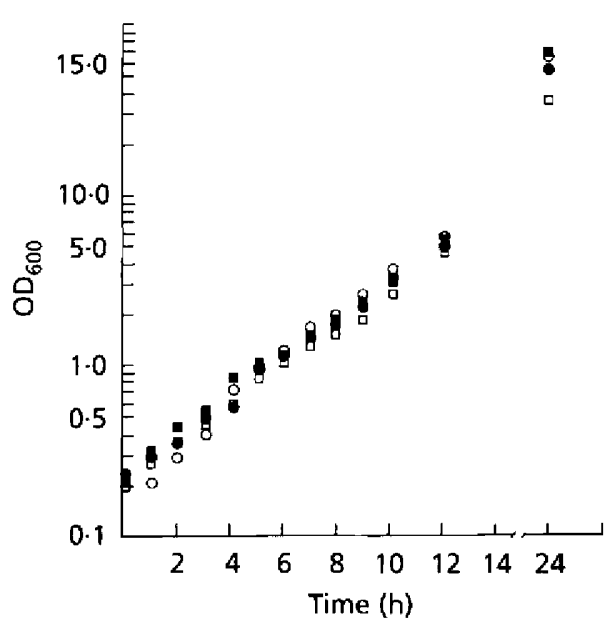

Fig. 2. Growth analysis of $C$. albicans strains CAl4, CFD1, CFD2 and CFD3. Strains were grown in YEPD supplemented with fatty acids. Overnight cultures of each strain were diluted into fresh medium and cultures were then incubated at $37^{\circ} \mathrm{C}$. Samples were removed at the indicated timepoints. O, CAl4; O, CFD1; $\square$, CFD2; $\square$, CFD3. The growth characteristics of CAl4, CFD1 and CFD3 when strains were grown without medium supplementation were identical to those depicted.

cassette reported by Fonzi \& Irwin (1993). The cassette was engineered to introduce a $984 \mathrm{bp}$ deletion in the condensing reaction domain of FAS2 as described in Methods. C. albicans CAI4 (Fonzi \& Irwin, 1993), a derivative of the clinical isolate C. albicans SC5314 (Gillum et al., 1984), served as the parental strain. The F $F S 2$ null mutant, designated CFD2, was generated by sequential disruption of each allele. Strain CFD3 contained a reconstructed $F A S 2$ allele and was included in subsequent analyses to ensure that phenotypic traits attributed to CFD2 were the result of mutation of $F A S 2$ and not due to other alterations that may have been introduced during CFD2 construction. In addition, since different $F A S 2$ alleles were disrupted in strains CFD1 and CFD3, any allelic effects of single disruption could be detected. Southern blot analysis confirmed the expected recombination patterns (Fig. 1). Briefly, genomic DNA of the CAI4 parent has two Bg/II fragments of 3.9 and $3.7 \mathrm{~kb}$ containing FAS2 sequences (Fig. 1, lane 1). CFD1 (Fig. 1, lane 2) showed disruption of a single $F A S 2$ allele in which two new $B g / \mathrm{II}$ fragments $(7.7$ and $3.1 \mathrm{~kb})$ were present along with the wild-type pattern of the undisrupted allele. Both F AS2 alleles of CFD2 have been altered (Fig. 1, lane 4 ), as shown by the disappearance of all wild-type allele BgIII fragments. Finally, one FAS2 allele has been reconstructed in CFD3 (Fig. 1, lane 5), demonstrated by the teestablishment of the original 3.9 and $3.7 \mathrm{~kb} \mathrm{Bg/II}$ fragments.

After construction, the growth characteristics of the strains were examined. CFD2 does not grow either in liquid or on solid medium without fatty acid supplementation, while strains CFD1 and CFD3 showed no such requirements. The growth rate of CFD1 and CFD3 in unsupplemented medium was essentially identical to that of the parental strain (Fig. 2). The growth rate of CFD2, 


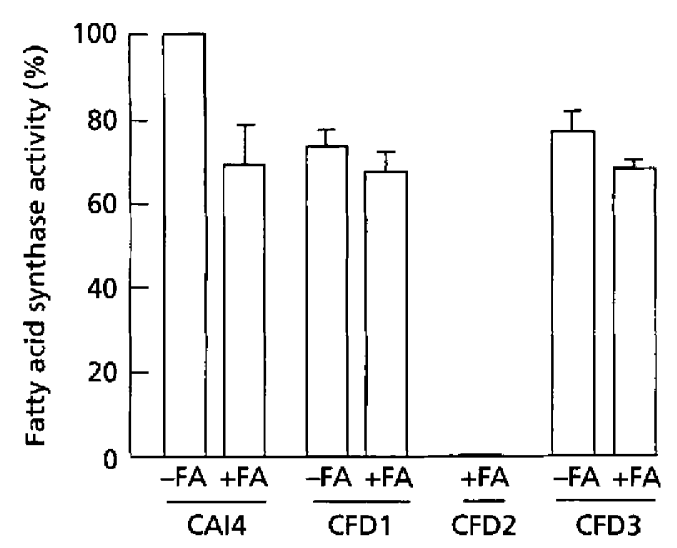

Fig. 3. Fatty acid synthase activity in C. albicans FAS2 mutants. The indicated strains were grown in YEPD medium lacking (-FA) or supplemented (+FA) with fatty acids. Data are expressed as a percentage of the fatty acid synthase activity in the parental strain (CAl4) grown in the absence of fatty acids.

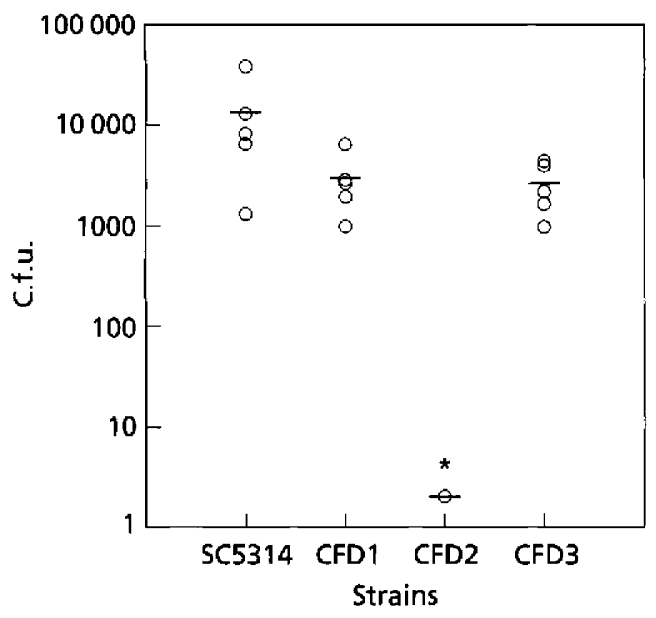

Fig. 4. Comparison of infection with C. albicans strains SC5314, CFD1, CFD2 and CFD3 in desalivated rats. Circles represent c.f.u. recovered from individual animals. The mean is designated by a horizontal bar. * $C$. albicans was observed in only two of the five animals infected with CFD2.

when provided with fatty acids, showed no significant differences from the wild-type (Fig. 2). The yeast-tohyphal transition of all mutant strains occurred normally when induced by temperature shift (data not shown).

\section{Fatty acid synthase activity of mutant strains}

In order to examine the effect of $F A S 2$ distuption on fatty acid synthase, the activity of the enzyme from all strains was assayed (Fig. 3). Strain CFD2 had no detectable fatty acid synthase activity when grown in medium supplemented with fatty acids, and the strain does not grow without such additions. Both strains disrupted in only a single $F A S 2$ allele (CFD1 and CFD3) maintained approxi- mately $80 \%$ of wild-type activity when grown on a medium without fatty acid supplementation. There was no significant difference in fatty acid synthase activity in cells grown in the presence of fatty acids in the case of $\mathrm{CAI} 4, \mathrm{CFD} 1$ or $\mathrm{CFD} 3$.

\section{Avirulence of CFD2 in the rat model of oropharyngeal candidiasis}

FAS2 mutant strains were tested for the ability to establish infection in the rat model of oropharyngeal candidiasis. The results depicted in Fig. 4 demonstrate that the ability of CFD2 to colonize the oral cavity is severely impaired. Extremely low numbers of CFD2 were recovered from only two of five inoculated animals. In contrast, CFD1 and CFD3 established infection and were readily recovered from animals at the termination of the experiment. The extent of colonization for these strains, however, was approximately fivefold lower than that observed for the parental strain SC5314. CAI4 was not used as a control in these experiments as it has been demonstrated previously that deletion of $U R A 3$ renders the strain avirulent in the rat model (Cole et al., 1995).

\section{DISCUSSION}

The results demonstrate that a $C$. albicans $F A S 2$ null mutant strain, CFD2, has been obtained. CFD2 was unable to grow without an exogenous supply of fatty acids, and fatty acid synthase was undetectable in the strain. However, the growth rate of CFD2, when supplied with fatty acids, was comparable to that of the parental strain. Finally, Southern blot analysis confirmed mutation of both $F A S 2$ alleles. In contrast, strains with disruption of either of the FAS2 alleles (CFD1 and CFD3), when grown without an exogenous fatty acid source, retained approximately $80 \%$ of wild-type fatty acid synthase activity. The growth rate of these strains was not adversely affected by this level of loss of enzyme activity. Addition of fatty acids to the growth medium resulted in only a slight reduction of enzyme activity in the case of strains disrupted in one allele of the FAS2 gene, in comparison to the approximately $20 \%$ drop in wild-type activity under the same conditions.

Strain CFD2 was not effective in establishing infection in the rat model of oropharyngeal candidiasis, indicating that fatty acid synthase activity is required for colonization and growth. No systematic studies concerning the level of fatty acids in the rat oral cavity have been reported, and thus it is not possible to speculate concerning the level of free fatty acids that might be available to mutant strains. Similarly, conclusions concerning the ability of CFD2 to colonize at other locations cannot be drawn from the results, and such information will come only from direct experiments. It is clear, however, that the presence of free fatty acids in the oral cavity or their supply through the activity of secreted phospholipases does not compensate for the lack of fatty acid synthase activity in CFD2. On the other hand, strains CFD1 and CFD3 were capable of promoting infection despite a $20 \%$ reduction in fatty acid 
synthase activity. Paradoxically, the number of c.f.u. recovered from rats infected with CFD1 and CFD3 was approximately fivefold less than noted for the wild-type despite the fact that no significant differences in the in vitro growth rate of mutant and parental strains were observed. It is, of course, possible that CFD1 and CFD3 have a slower in vino growth rate, which would account for reduced tecovery. Alternatively, it is possible that initial colonization does not occur as efficiently with these two strains.

Most importantly, the results suggest that fatty acid synthase may be a valid target for the develnpment of antifungal agents. While the enzyme is ubiquitous, significant structural differences exist between the mammalian and fungal enzyme, suggesting that compounds with differential activity might be found. In this regard, the effect of modifications to cerulenin, an antilipogenic agent that specifically and irreversibly inhibits fatty acid synthase by inhibition of the condensing reaction (Omura, 1981), on activity of the human and C. albicans enzyme was investigated (Broedel et al., 1996). Alterations including halogen, methoxy or trifluoromethyl group decoration of the cerulenin molecule resulted in inhibitory activity against fungal fatty acid synthase, but no detectable activity against the human counterpart. Similar results were noted with a compound containing an ether linkage between the terminal phenyi group and the keto-epoxide moiety of phenylcerulenin (Broedel et al., 1996). In addition, preliminary daca presented in the same study detected three as yet unidentified natural products showing differential inhibitory activity. The validation of fatty acid synthase as a potential target in the oropharyngeal model should lead to studies to test the in vivo efficacy of agents that are active against fatty acid synthasc. The rcsults do not provide evidence that fatty acid synthase will be a suitable target in other models of candidiasis or for infections mediated by other fungi. Experiments are in progress that will address whether fatty acid synthase will be a target of general significance.

\section{ACKNOWLEDGEMENTS}

We wish to thank Ms Sylvia Pearson for her assistance in experiments utilizing the rat model of oropharyngeal candidiasis, and Ms Patricia Sura for help in figure preparation. We are grateful to $\mathrm{Dr}$ William Fonzi for providing C. albicans strains SC5314 and CAl4, and the plasmid p5921. This work was supported in part by Chek Tec Corp. and by Public Health Service Grants DE00189 and PO1 AI37251 from the National Institutes of Health.

\section{REFERENCES}

Becker, J. M., Henry, L. K., Jiang, W. \& Koltin, Y. (1995). Reduced virulence of Candida albians mutants affected in multidrug resistance. Infect Immun 63, 45284530 .

Boeke, J. D., Lacroute, F. \& Fink, G. R. (1984). A positive selection for mutants lacking orotidine-5'phosphate decarboxylase activity in yeast: 5 fluoro-orotic acid resistance. Mol Gen Genet 197, 345346
Broedel, S. E., Jr, Zhao, X.J. \& Cihlar, R. L. (1996). Fatty acid FL L44 synthasc as a target in the development of new antifungals. Recent Res Det: Antimicrob Agents Chemother 1, 25-33.

Bulawa, C. E., Miller, D. W., Henry, L. K. \& Becker, J. M. (1995). Attenuated virulence of chitin-deficient mutants of Candida albicans. Proc Natl Acad Sci USA 92, 10570-10574.

Cole, M. F., Bowen, W. H., Zhao, X.-J. \& Cihlar, R. L. (1995). Avirulence of Candida albicans auxotrophic mutants in a rat model of oropharyngeal candidiasis. FEMS Microbiol Lett 126, 177-180.

Cutler, J. E. (1991). Putative virulence factors of Candida albicans. Annu Rev Microbiol 45, 187-218.

De Bernardis, F., Adriani, D., Lorenzini, R., Pontieu, E., Carruba, G. \& Cassone، A. (1993). Filamentous growth and elevatcd vagino pathic portential of a nongerminative variant of Candida albicans expressing low virulence in systemic infection. Infect Immun 61, $1500-1508$

Fonzi, W. \& Irwin, M. Y. (1993). Isogenic strain construction and gene mapping in Candida albicans. Genetics 134, 717-728.

Ghannoum, M. A., Spellberg. B., Saporito-Irwin, S. M. \& Fonzi, W. F. (1995). Reduced virulence of Candida albicans PHR 1 mulants. Infot lmmin 63, 45284530 .

Gillum, A. M., Tsay, W. Y. H. \& Kirsch, D. R. (1984). Isolation of the Candida albicans gene for orotidine- $5^{\prime}$-phosphate decarboxylase by complementation of $S$. cerevisiae ura 3 and E. coli pyrF mutations. Mol Gen Gentet 198, 179-182.

Holmberg, K. \& Meyer, R. D. (1986). Fungal infections in patients with AIDS and AIDS-related complex. Sicand I Infert Dis 18, $179-185$.

Kuziora, M. A., Chalmer, J. H., Jr, Douglas, M. G., Hitzeman, R. A., Mattick, J. S. \& Wakil, S. J. (1983). Molecular cloning of fatty acid synthctase genes from Sacharomyces cerevisiae. I Biol Cbem 258, $11648-11653$.

Lynen, F. (1969). Yeast fatty acid synthase. Methods Enzymol 14, $17-33$.

McElhaney-Feser, G. E. \& Cihlar, R. L. (1994). Purilication and characterization of fatty acid synthase from Candida albicans, strain 4918 , and two derived spontaneous cerulenin-resistant mutants. $J$ Med Vet Mrol 32, 13-20.

Meitner, S. W., Bowen, W. H. \& Haidaris, C. G. (1990). Oral and esophageal Candida albicans infection in hyposalivatory rats. Infect Immun 58, 2228-2236.

Odds, F. C. (1988). Candida and Canditosis, London: Baillière Tindall.

Ōmura, S. (1981). Cerulenin. Methods Enzymol 72, 520-532.

Ruchel, R., De Bernardis, F., Ray, T. L., Sullivan, P. A. \& Cole, G. T. (1992). Candida acid proteinases. J Med V et Mycol 29 (Suppl.), 1-9.

Sambrook, J., Fritsch, E. F. \& Maniatis, T. (1989). Molecular Cloning : a Laboratory Manual. Cold Spring Harbor, NY: Cold Spring Harbor Laburatory.

Schweizer, E., Müller, G., Roberts, L. M., Schweizer, M., Rösch, J., Weisner, P., Beck, J., Stratman, D. \& Zauner, I. (1987). Genetic control of fatty acid synthetase biosynthesis and structure in lower fungi. Fai Sci Technol 89, 570-577.

Sherman, F., Fink, G. R. \& Hicks, J. B. (1986). Laboratory Course Manual for Methods in Yeast Genetics. Cold Spring Harbor, NY: Cold Spring Harbor Laboratory.

Siggaard-Anderson, M. (1993). Conserved residues in condensing enzyme domains of fatty acid synthases and related sequences. Protein Seq Data Anal 5, 325-335. 
Smith, S. (1994). The animal fatty acid synthase: one gene, one polypeptide, seven enzymes. FASEB J 8, 1248-1259.

Soll, D. R. (1992). High frequency switching in Candida albicans. Clin Microbiol Rev 5, 183-203.

Stoops, J. K., Awad, E. S., Arslanian, M. J., Gussberg. 5. \& Wakil, S. J. (1978). Studies on the yeast fatty acid synthetase: subunit composition and structural organization of a large multifunctional enzyme complex. $J$ Biol Chem 253, 4464-4475.
Southard, S. B. \& Cihlar, R. L. (1995). Analysis and expression of the Candida albicans F AS2 gene. Gene 156, 133-138.

Witkowski, A., Rangan, V. S., Randhawa, Z. I., Amy, C. M. \& Smith, S. (1991). Structural organization of the multifunctional animal fatty-acid synthase. Eur J Biochem 198, 571-579.

Received 23 February 1996; revised 22 May 1996; accepted 24 May 1996. 